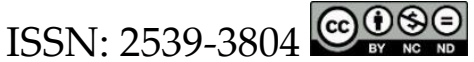

\title{
Estrategias léxicas de ingenieros para el contexto lector ${ }^{1}$
}

\author{
Martha Isabel Hernández Pastuzano \\ Renfrew Educational Services \\ Calgary, Canadá \\ Fanny Hernández Gaviria \\ Universidad del Valle \\ Cali, Colombia
}

\section{Resumen}

Este artículo muestra los resultados de un estudio de caso con un grupo de estudiantes de ingeniería en una universidad pública de Colombia. El objetivo del estudio se centró en explorar las estrategias léxicas usadas por los estudiantes, su frecuencia de uso y su grado de efectividad. La información se recolectó a partir de talleres de lectura y de reflexiones orales sobre los procesos seguidos en la interacción con textos de su área profesional en inglés, particularmente en relación con el componente léxico. Los resultados de esta investigación indican que los estudiantes participantes conocen y utilizan un repertorio amplio de estrategias para interpretar el componente léxico presente en los textos. Sin embargo, las estrategias usadas con mayor frecuencia no son siempre las más efectivas y las que usan más efectivamente son usadas con poca frecuencia y por pocos estudiantes. Los resultados de la investigación son una buena base para pensar en una propuesta de intervención que busque mejorar los procesos de comprensión de lectura en comunidades académicas del área de ingeniería.

Palabras clave: estrategias léxicas; ESP; comprensión lectora; lenguaje académico.

\footnotetext{
${ }^{1}$ Este trabajo investigativo se llevó a cabo en el marco de la Medida Transitoria declarada a partir de la Resolución No. 084 de noviembre 4 de 2016, que facultó al Consejo Académico para reglamentar de manera transitoria el reingreso a los Programas de Posgrado a las personas que estuvieron vinculadas a la Universidad en calidad de estudiantes regulares de Posgrado. El trabajo se suscribió a nombre de Martha Hernández, estudiante del programa de Maestría en Lingüística y Español de U.V. con el título Estrategias léxicas en ESP usadas por estudiantes de ingeniería eléctrica y dirigido por Fanny Hernández, profesora de la Escuela de Ciencias del Lenguaje en la Universidad del Valle.
} 


\section{Abstract \\ Lexical strategies of engineers for the reader context}

This article shows the results of a case study with a group of engineering students in a public university in Colombia. The aim of the study was to explore the lexical processing strategies used by this group of students, the frequency of use, and its effectiveness. Data was collected through reading comprehension workshops and think-aloud protocols, in which students reported the steps they followed to cope with unknown words when reading English texts from their field. Results showed that the participants in the study know and make use of a wide range or strategies to interpret new words when reading professional texts. However, the most frequently used strategies are rarely the most effective and those recognized as the most effective are seldom used by a few students. These results might be a good source for the design and development of teaching units aiming at improving reading comprehension in students of the engineering area.

Key words: lexical processing strategies; ESP; reading comprehension; academic language.

\section{Résumé}

\section{Stratégies lexicales des ingénieurs pour le contexte du lecteur}

Cet article présente les résultats d'une étude de cas mené avec un groupe d'étudiants de génie dans une université publique de Colombie. L'objectif de cette recherche et d'explorer les stratégies lexicales employées par les étudiants, leur fréquence d'utilisation et leur degré d'efficacité. L'information a été collectée à partir des ateliers de lecture et des réflexions orales sur des processus d'interaction avec des textes de leur discipline en anglais, particulièrement, avec un rapport avec la composante lexicale. Les résultats de travail indiquent que les participants connaissent et se servent d'un répertoire large de stratégies afin d'interpréter le lexique présent dans les textes. Néanmoins, les stratégies les plus employées ne sont toujours pas les plus efficaces et celles qu'ils utilisent de manière plus effective, sont moins employées par très peu d'étudiants. Les données de cette étude se constituent en tant que base pour penser à une proposition d'intervention visant à améliorer les processus de compréhension en lecture au sein des communautés académiques dans le domaine du génie.

Mots-clés : stratégies lexicales ; ESP ; compréhension en lecture ; langage académique. 


\section{CóMO CITAR ESTE ARTÍCULO}

Hernández Pastuzano, M. I., y Hernández Gaviria, F. (2019). Estrategias léxicas de ingenieros para el contexto lector. Lenguaje, 47(2), 379-408. doi: 10.25100/lenguaje.v47i2.6998 


\section{INTRODUCCIÓN}

El idioma Inglés es usado hoy en día en la mayoría de los contextos profesionales, su demanda sigue creciendo irremediablemente en el ámbito educativo y esta tendencia es observable en el incremento evidente de cursos de inglés en las grandes y pequeñas ciudades del mundo. Este requerimiento al ciudadano de hoy tiene su efecto también en el ámbito educativo; todos los programas de educación básica, media y superior se han visto en la necesidad de revisar sus currículos para adaptarlos y crear contextos de aprendizaje que favorezcan el desarrollo del conocimiento de esta lengua extranjera. En el nivel de educación superior los programas profesionales buscan que sus estudiantes desarrollen, durante su periodo de formación, un nivel de lengua que les posibilite acceder a conocimiento especializado para que se desempeñen de manera eficiente en su vida profesional futura. Sin embargo, a pesar de los cambios y los requerimientos reales del profesional de hoy, las políticas nacionales o institucionales no siempre generan las condiciones de aprendizaje necesarias para alcanzar los niveles deseados y este hecho asigna al docente un papel protagónico en la formación de este profesional.

En este contexto de aprendizaje, y en el marco del cubrimiento de estos requerimientos, es indispensable instruir explícitamente en el manejo de estrategias de lectura en esta lengua extranjera. Esta afirmación se fundamenta en múltiples estudios, tales como el de Barbera (2011), quien investigó las estrategias usadas por un grupo de estudiantes para descifrar el significado de palabras en textos académicos. El estudio concluye que es necesario integrar a los programas de los cursos de lengua extranjera el desarrollo de nuevas estrategias o, en su lugar, mejorar el uso de las que ellos ya utilizan. En la misma línea, Hassan y Abubakr (2015) buscaron identificar la forma como estudiantes kurdos de la Universidad de Sulaimani, localizada en Irak, aprendían vocabulario en inglés. Los investigadores construyeron la hipótesis descriptiva de que las estrategias usadas por los estudiantes no eran promovidas en el contexto universitario y, además, que los estudiantes no eran conscientes de la importancia de la comprensión y el manejo de vocabulario en la búsqueda de logros académicos en esta lengua.

En el contexto colombiano y particular de la Universidad del Valle, el aprendizaje del inglés como lengua extranjera se hace a través de la asignatura "Lectura de textos académicos en inglés" en todos los planes de estudio, exceptuando el programa de Lenguas Extranjeras. El objetivo principal de esta asignatura es crear un escenario apropiado para que los estudiantes desarrollen estrategias, fundamentalmente de comprensión de lectura, que les permitan interactuar con textos auténticos en inglés, en el marco de su área profesional. La asignatura es reconocida en el contexto de la Universidad del Valle con la sigla ESP, Inglés con propósitos específicos (English for Specific Purposes) y está organizada en dos o en cuatro niveles. El reconocimiento del importante aporte de esta área en la formación de todos los 
profesionales de la Universidad ha generado en los profesores de los cursos de ESP un alto compromiso y un interés investigativo que propende por mejoramiento continuo (Hernández, 2008; Lakatos, 2004; Ortiz, 2011).

Las circunstancias descritas despiertan en la autora de este trabajo, y docente a cargo de cursos de ESP por un tiempo en la Universidad, el interés particular de explorar y describir las estrategias usadas por un grupo de estudiantes de ingeniería eléctrica para interpretar el componente léxico presente en textos en inglés de su área profesional. El interés específico por este aspecto responde a que los estudiantes lo perciben como un elemento que les representa un alto grado de dificultad, percepción que es corroborada por la teoría de ESP en la que se sugiere dar atención especial al componente léxico en el diseño de los programas de esta asignatura (Dudley-Evans y St. John, 1998). La identificación de las estrategias utilizadas por los estudiantes para comprender el componente léxico en los textos y el grado de efectividad logrado con su uso contribuiría a definir las estrategias que requieren instruirse en estos cursos para dar mayor efectividad a los procesos de enseñanza y aprendizaje del inglés en este contexto universitario.

La investigación llevada a cabo es un estudio de caso de corte cualitativo, complementado con elementos de referencia porcentual y de frecuencia. El grupo de estudiantes de ingeniería eléctrica participante estaba matriculado en nivel II de "Lectura de textos académicos en inglés". El curso estaba conformado por estudiantes que cursaban su primer o segundo año de carrera; el corpus recogido corresponde a la información provista por nueve estudiantes en segundo año; se tuvo en cuenta que este grupo había tenido un acercamiento mayor a contenidos de su área profesional. La recolección de la información se hizo en doce semanas, en las que se desarrollaron nueve talleres en modalidad diversa: selección múltiple, pregunta abierta, protocolos de voz alta e inferencia de significado y apareamiento con la estrategia usada.

El análisis de la información permitió, en primer lugar, identificar las diferentes estrategias que usan los estudiantes para comprender el léxico en textos de su área profesional; en segundo lugar, definir las estrategias que son usadas con mayor y menor frecuencia y efectividad en los procesos de lectura. Así mismo, se reconoció que el estudiante se asume como participante activo en los procesos de interacción con los textos; esto se puede observar en las posturas que adoptan frente a procesos cognitivos y meta-cognitivos, inherentes a este tipo de interacción. Estos descubrimientos fueron la base para establecer las relaciones más significativas entre las posibilidades y las dificultades de los estudiantes para abordar textos de su área profesional y postular, con esta base, una propuesta inicial para definir una intervención en estrategias de aprendizaje que dinamice el uso de estrategias léxicas y mejore la comprensión de textos del área profesional. 


\section{FUNDAMENTACIÓN TEÓRICA}

La revisión de los fundamentos de la enseñanza de ESP permite entender por qué y cómo son planteados los cursos de Lectura de textos académicos; la conceptualización del lenguaje científico y la comprensión de algunos rasgos gramaticales y léxicos particulares en los textos académicos permite reconocer los aspectos que ofrecen dificultad a los estudiantes en su proceso de aprendizaje del inglés como lengua extranjera. De igual forma, sus dificultades de lectura se comprenden mejor con un análisis contextualizado de las características de este tipo de texto, en relación con los principios teóricos que respaldan el énfasis en el género expositivo. Por otro lado, se requiere estudiar las estrategias de aprendizaje, tanto su papel en el proceso cognitivo como su influencia en la formación de un aprendiz exitoso.

\section{Surgimiento del Inglés para propósitos específicos (English for Specific Purposes- ESP)}

Hutchinson y Waters (1987) enumeran tres razones que impulsaron el surgimiento de ESP: las necesidades creadas por la expansión científica, técnica y económica a nivel mundial después de la segunda guerra mundial, un cambio fuerte en la lingüística y un cambio en la psicología educativa. El poder económico de los Estados Unidos después de la guerra hizo que el inglés se tornara en el idioma internacional de dominio en la tecnología y el comercio, de manera que saber inglés dejó de ser una cuestión de placer o prestigio y se convirtió en la puerta de acceso a la ciencia y a la economía del momento. De allí que surgió una nueva generación de aprendices con intereses y necesidades particulares, que llevaron a que la enseñanza del inglés tuviera un propósito definido y a que se iniciara un proceso de revisión de la manera de abordar su enseñanza:

(...) La perspectiva de que el inglés que un grupo particular de aprendices necesitaba podía ser identificado analizando las características lingüísticas de su área de especialidad de estudio o trabajo tomó fuerza. El principio guía de ESP fue "dime para qué necesitas inglés y yo te diré la clase de inglés que necesitas". (Hutchinson y Waters, 1987, p. 8). ${ }^{2}$

La revisión de los programas de cursos llevó a incluir textos del área de especialización de los aprendices en los cursos de inglés; se pensó que si el contenido era relevante para el estudiante, su motivación mejoraría y por ende el aprendizaje. Kennedy y Bolitho (1984) reconocieron en esta orientación un énfasis en el aprendiz, un

\footnotetext{
2 Esta y todas las traducciones de originales en inglés son hechas por las autoras de este artículo.
} 
factor decisivo en el aprendizaje del inglés. En este desarrollo se reconocen tres razones que impulsaron el surgimiento de ESP: expansión y demanda del inglés, cambios en el campo de la lingüística y enfoque centrado en el aprendiz.

\section{Características del ESP}

Dudley-Evans y St. John (1998) plantean características absolutas y variables:

\section{Características absolutas:}

- $\quad$ ESP está diseñado para cumplir con las necesidades específicas del aprendiz.

- ESP hace uso de la metodología y de las actividades que subyacen a la disciplina.

- ESP hace énfasis en aspectos del lenguaje (gramática, vocabulario, registro), habilidades, discurso y género característico de la disciplina.

\section{Características variables:}

- $\quad$ ESP puede ser diseñado para una disciplina específica.

- $\quad$ ESP puede usar una metodología diferente de la usada para enseñar inglés general.

- ESP, generalmente, es diseñado para aprendices adultos en un nivel profesional.

- ESP, generalmente, es diseñado para estudiantes con niveles intermedio o avanzado de lengua, aunque podría enseñarse a personas con nivel inicial.

Strevens (como se citó en Gatehouse, 2001) plantea características absolutas y variables muy similares en las que hace énfasis el ESP para atender las necesidades del aprendiz. Sin embargo, Strevens adiciona dos (una absoluta y una variable) que Dudley-Evans y St. John (1998) no consideran:

- $\quad$ ESP está en contraste con inglés general.

- $\quad$ ESP puede ser restringido a una habilidad del lenguaje, por ejemplo, la lectura.

La definición del área de ESP puede ser ampliada con las concepciones formuladas por autores tales como Hutchinson y Waters (1987), quienes afirman que las decisiones relacionadas con el contenido y la metodología se basan en las razones para aprender. Por su parte, Kennedy y Bolitho (1984) aseveran que ESP fue la respuesta a quienes ya tenían conocimiento de inglés y eran conscientes de su propósito de aprendizaje. Robinson (1980) plantea que ESP debe suplir las necesidades de quienes requieren del 
idioma antes de asumir un papel profesional. Estos autores coinciden en que los aprendices saben qué les motiva a estudiar la lengua.

\section{Género Expositivo}

El género imperante en las ciencias es el expositivo. Los textos pertenecientes a este género proveen datos de una forma educativa y con un propósito definido. Estos textos deben ser claros, concisos y poseen una estructura organizada que facilita la comunicación de la información que promueve la comprensión de lectura. El conocimiento de la estructura de este tipo de texto es reconocida como una estrategia eficiente para promover la comprensión de lectura (Zarrati, Nambiar y Tg. Mohamad Maasum, 2014). Esta afirmación coincide con Hyland (2006), quien plantea que la familiarización con los textos de un género específico facilita la comprensión, puesto que el lector que conoce la estructura del texto puede anticipar la información que se va a presentar. Cuando se desconoce la estructura del texto se está en desventaja porque ello impide desarrollar un plan de lectura (Meyer, Brandt y Bluth como se citó en Akhondi, Malayeri y Samad, 2011).

\section{Autenticidad de los Textos}

Nunan (1988) define los materiales auténticos como los que han sido producidos con un fin diferente al de enseñar una lengua. De acuerdo con McGrath (2002), la autenticidad de los textos es importante porque les da a los aprendices una muestra del mundo real. Sin embargo, se plantea la posibilidad de hacer consideraciones pedagógicas tales como mejorar la calidad de impresión, ajustar su extensión, siempre y cuando no se afecte su autenticidad. McGrath (2002) propone algunos criterios para la selección de textos auténticos: relevancia en el contexto, interés intrínseco del tema, respeto a la cultura del lector, exigencias lingüísticas, exigencias cognitivas, consideraciones logísticas (extensión, legibilidad), calidad (con relación al género) y utilidad.

\section{Lenguaje científico - académico y sus características}

Hutchinson y Waters (1987) sostienen que los lenguajes de especialidad para escribir sobre ciencia se remontan a los años sesenta y a principios de los setenta, y lo asocian con el trabajo de Strevens en 1964 y Ewer y Latorre en 1966, quienes contrastan los registros específicos con los registros del inglés general e identifican los rasgos gramaticales y léxicos del registro específico profesional. En sus estudios, Ewer y Latorre (como se citó en Hutchinson y Waters, 1987) encontraron que el registro del inglés científico presenta alta frecuencia de formas pasivas, compuestos nominales y de presente simple. Este principio hizo que se hiciera énfasis en el análisis del registro. Posteriormente se presentaron argumentos opuestos, expertos en la enseñanza 
coinciden en afirmar que hay unas formas más usadas en unos contextos que en otros y que el contexto científico tiene características particulares que no pueden ser ignoradas. $\mathrm{Al}$ respecto, Hyland (2004) agrupa los rasgos del lenguaje académico en tres áreas:

- Alta densidad léxica, una alta proporción de palabras de contenido en relación con palabras gramaticales como preposiciones, artículos y pronombres. Ejemplo: Investment in a rail facility implies a long-term commitment" vs. "If you invest in a rail facility this implies that you are going to be committed for a long term (Halliday y Hasan como se citó en Hyland, 2006, p. 145).

- Alta presencia del estilo nominal: Las acciones y eventos se presentan como sustantivos más que como verbos. Ejemplo: Water boils at $100 \mathrm{C}$ degress $=$ water boiling point is 100. Las relaciones entre las entidades se hacen a partir de transformaciones de procesos en objetos.

- Construcciones impersonales: Los pronombres de primera persona son remplazados por formas pasivas: "The solution was heated", presencia del sujeto "it": "it was posible to measure the pieces" y el uso de personalización (objeto con papel de agente), conocido como "personalización": "The data suggests".

\section{Características del léxico usado en los textos científicos}

En la caracterización del léxico en los textos científicos, Trimble (1985) propone una agrupación en tres áreas léxicas: vocabulario técnico, vocabulario sub-técnico y compuestos nominales. Swales (1983) y Bhatia (1993) consideran que los compuestos nominales no forman parte del léxico sino de los rasgos gramaticales del discurso científico. Este trabajo adopta su planteamiento:

- Vocabulario Técnico: Este vocabulario recoge el conjunto de términos especializados que se utilizan en una disciplina determinada y hacen parte intrínseca del aprendizaje del área de estudio. A manera de ilustración, un estudiante de ingeniería eléctrica estudiando "analog instruments" encontrará los conceptos de dynamometer, torque, ampere-turns en su proceso de lectura; estos términos pertenecen al léxico técnico de esa área.

- Vocabulario sub-técnico: Este vocabulario es el de mayor interés por parte de expertos en el área; este vocabulario está constituido por todas las palabras que no son específicas a una disciplina, pero aparecen con regularidad en textos técnicos y científicos. Por ejemplo, intense, reflection, accumulate, tendency, isolate, etc. Kennedy y Bolitho (1984) argumentan que la ocurrencia de este vocabulario es de más o menos un $80 \%$ en un texto y que, por lo tanto, debe asignársele prioridad en un programa de enseñanza de lengua extranjera. 
Dada la importancia de este vocabulario, Beck, McKeown y Kucan (2013) lo presentan en tres categorías: Tier I, constituido por las palabras que se usan en conversaciones cotidianas, por ejemplo: "run, look"; Tier II, palabras de alta frecuencia, generalmente usadas en más de una disciplina de estudio, pero no llegan a ser técnicas; los autores las llaman palabras "educated", por ejemplo: "scamper and scrutinize"; Tier III, palabras técnicas que se inscriben dentro de una disciplina de estudio específica, por ejemplo: "blatosphere", "igneous". Desde esta perspectiva, Tier II y Tier III constituyen el vocabulario académico y son, de hecho, las bases del contexto escolar norteamericano para potenciar el éxito de los estudiantes en comprensión de lectura.

\section{Estrategias de aprendizaje en segunda lengua}

El interés en las estrategias de aprendizaje para una segunda lengua tomó fuerza en los años setenta cuando se consideró esencial no sólo centrarse en el proceso de enseñanza, sino en el aprendizaje y reconocer al estudiante como participante activo. Esta concepción tiene un efecto determinante en el proceso de aprendizaje de una segunda lengua. O'Malley y Chamot (1990) reconocen como pioneros en este campo a Rubin y a Naiman, quienes identificaron y clasificaron las estrategias que los estudiantes reportaban o que ellos mismos identificaban en sus aulas.

O'Malley y Chamot (1990) reconocieron que las clasificaciones iniciales permitieron demostrar que los estudiantes poseen un variado número de procedimientos que les ayuda a ser efectivos en el aprendizaje. En este reconocimiento O'Malley y Chamot (1990) definen las estrategias de aprendizaje como "Los pensamientos y comportamientos que los individuos usan para ayudarse a comprender, aprender o retener nueva información" (p. 1). Ellos sustentaron que un aprendiz exitoso usa estrategias especiales que pueden ser instruidas en el contexto académico.

Su clasificación es bien reconocida en el campo de las estrategias:

- Estrategias Metacognitivas: sirven para monitorear la eficiencia en los procesos de aprendizaje y de uso de una lengua y ayudan a tomar decisiones en los procesos.

- Estrategias Cognitivas: involucran la manipulación de información y las actividades que se emprenden para adquirir o retener información.

- Estrategias Sociales / afectivas: se asocian a las relaciones interpersonales y de control de las emociones del aprendiz.

Otro sistema de clasificación reconocido, y considerado muy completo, es el de Oxford (1990), quien define las estrategias de aprendizaje como "acciones específicas que toman los aprendices para hacer el aprendizaje más fácil, más rápido, más divertido, más centrado en ellos, más afectivo y más fácil de transferir a otras situaciones" (p. 8). Su categorización incluye dos grandes grupos: estrategias directas e 
indirectas. En las directas están las cognitivas, las de memoria y las de compensación. En las indirectas están las metacognitivas, las afectivas y las sociales.

\section{Estrategias de comprensión de lectura}

Entendiendo que un propósito de lectura puede variar de un lector a otro, de acuerdo con su requerimiento, lectura detenida, lectura global o de selección de material, es importante desarrollar en los estudiantes estrategias que den respuesta a sus requerimientos de lectura para que puedan así dar cumplimiento a sus objetivos académicos. Dos estrategias consideradas útiles en toda actividad de lectura en lengua extranjera son: identificación del contexto y deducción de significados; en el contexto académico particular, el proceso de comprensión de lectura se puede favorecer, complementariamente, con el desarrollo de tres tipos de estrategias: estrategias previas, estrategias simultáneas y estrategias posteriores a la lectura. Las primeras centran su atención en activar los conocimientos previos y en predecir el objetivo del texto; las segundas buscan adentrarse en el sentido del texto, incrementando el vocabulario, y las terceras promueven la reflexión, el análisis y la síntesis de los contenidos del texto. El reconocimiento del vocabulario general y el específico es esencial en la aplicación de estrategias simultáneas a la lectura; este reconocimiento posibilita la identificación de las ideas principales del texto y la definición de las relaciones que se establecen entre ellas. Es decir, posibilitan la comprensión de los contenidos de un texto.

Teniendo en cuenta la relevancia de este tipo de estrategia, relacionada con el reconocimiento de los diversos niveles léxicos que ofrece un texto y su utilidad en la comprensión del texto, este trabajo profundiza en las particularidades de la estrategia léxica (Durán Escribano, 2001).

\section{Estrategias de comprensión léxica}

De acuerdo con Schmitt (1997), en el proceso de identificar y categorizar las estrategias del lenguaje, muchos estudios de los años ochenta y noventa estuvieron indirectamente relacionados con estrategias específicamente aplicables al aprendizaje del vocabulario. Sin embargo, muy pocas estrategias de vocabulario se estudiaron en profundidad (Schmitt, 1997). En los años dos mil surgió un mayor número de investigadores interesados en explorar las estrategias utilizadas por los estudiantes para inferir significados de palabras desconocidas: Nassaji (2003), Carlo et al. (2004), Barbera (2011), Yin (2010), Wang (2011), Graves y Fitzgerald y De Luca y Hummel (citados en Barbera, 2011). De estos estudios surgieron propuestas tales como la de Schmitt (1997), quien las divide entre actividades léxicas útiles para el descubrimiento inicial del significado de una palabra y actividades léxicas para recordar la palabra. La primera categoría, la denomina estrategias de descubrimiento (discovery strategies) y está estrechamente 
relacionada con el propósito de esta investigación. Las estrategias de descubrimiento las subdivide en dos categorías: estrategias de determinación (DET) y estrategias sociales (SOC). Las primeras requieren usar conocimiento lingüístico, claves del contexto, y las segundas involucran solicitar ayuda de alguien que se cree que posee el conocimiento (Schmitt, 1997).

Estrategias de determinación (DET)

- Analizar la función gramatical en la oración

- Analizar afijos y raíces

- Revisar si es un cognado en L1

- Analizar presencia de alguna imagen o gesto disponible

Estrategias sociales (SOC)
- Adivinar usando el contexto textual - Usar el diccionario bilingüe

- Usar el diccionario monolingüe

- Usar lista de palabras

- Usar tarjetas con palabras

- Pedir al profesor traducir a L1

- Pedir al profesor que parafrasee y dé un sinónimo de la palabra nueva

- Pedir al profesor que diga una oración incluyendo la palabra nueva

- Preguntarle el significado a un compañero

- Descubrir el significado de la nueva palabra a través de actividades grupales

Schmitt (1997) afirma que cuando un aprendiz no sabe el significado de una palabra, generalmente busca descifrarlo haciendo uso de estrategias de determinación tales como cognados en L1, inferencia a partir del contexto, uso de material de referencia o análisis de raíces o afijos de la palabra. Con relación a los cognados, se puede decir que son un excelente recurso, tanto para inferir el significado como para recordar palabras nuevas. Sin embargo, es importante tener en cuenta que estas interconexiones, basadas en similitudes de forma y significado entre lenguas, que llevan a generalizaciones, pueden convertirse en falsos cognados cuando se cae en una sobregeneralización. Una palabra puede tener la misma etimología en dos lenguas, pero haber evolucionado de manera diferente en ellas; desconocer esta evolución diferenciada puede afectar la comprensión de un texto (Durán Escribano, 2004).

Otra manera de descifrar el significado de una palabra nueva es el uso de la estrategia social: preguntarle a alguien que se espera que conozca el significado. Cuando se acude al docente, éste debe dar información traduciendo, dando un sinónimo, parafraseando con una definición, usando la palabra en contexto o combinando varias posibilidades. En complemento se sugiere promover el trabajo cooperativo (Schmitt, 1997). 


\section{Metodología}

La investigación corresponde a un estudio de caso (Yin como se citó en Brown, 2008). La muestra de análisis se construyó con un grupo de nueve estudiantes de ingeniería eléctrica en su segundo año de profesionalización. En este momento de la carrera ya se cuenta con un nivel de conocimiento de la disciplina de estudio y es posible trabajar textos de nivel intermedio de especialización académica. En primer lugar, se determinó el nivel de inglés de los participantes, a partir de dos talleres de comprensión de lectura y se les presentó el objetivo del proyecto de investigación. La definición de los libros fuente se hizo en coordinación con un profesional del área: Power System Analysis y Fundamentals of Electric Circuits, y la revista: ASU, Arizona State University. De estas fuentes se extrajeron los textos de trabajo, considerando exigencias lingüísticas y cognitivas, y el nivel de explotación del texto. La selección final se concretó en nueve textos. Con esta base se diseñaron 12 talleres de comprensión de lectura en diversas modalidades que permitían observar y dar cuenta de las estrategias usadas por los estudiantes para interpretar el significado del vocabulario nuevo y, posteriormente, evaluar su efectividad en el proceso de lectura, como se presenta en la Tabla 1.

Tabla 1. Selección de Lecturas

\begin{tabular}{|l|l|l|l|}
\hline $\begin{array}{c}\mathbf{N} \\
\circ\end{array}$ & \multicolumn{1}{|c|}{ Título } & \multicolumn{1}{c|}{ Fuente } & Modalidad de Taller \\
\hline $\mathbf{1}$ & $\mathbf{1 8 8 4}$ Exhibition & $\begin{array}{l}\text { Fundamentals of } \\
\text { Electric Circuits }\end{array}$ & Selección múltiple \\
\hline $\mathbf{2}$ & Problem Solving & $\begin{array}{l}\text { Fundamentals of } \\
\text { Electric Circuits }\end{array}$ & Selección múltiple \\
\hline $\mathbf{3}$ & What is Electrical Engineering? & $\begin{array}{l}\text { ASU, Arizona State } \\
\text { University }\end{array}$ & Selección múltiple \\
\hline $\mathbf{4}$ & Nuclear Power Plant & $\begin{array}{l}\text { Power System } \\
\text { Analysis }\end{array}$ & Pregunta abierta \\
\hline $\mathbf{5}$ & $\begin{array}{l}\text { Transmission and Distribution } \\
\text { System }\end{array}$ & $\begin{array}{l}\text { Power System } \\
\text { Analysis }\end{array}$ & Pregunta abierta \\
\hline $\mathbf{6}$ & Analog instruments & $\begin{array}{l}\text { Power System } \\
\text { Analysis }\end{array}$ & $\begin{array}{l}\text { Protocolos en voz alta } \\
\text { de interpretación léxica }\end{array}$ \\
\hline $\mathbf{7}$ & $\begin{array}{l}\text { Permanent-magnet moving coil } \\
\text { instruments }\end{array}$ & $\begin{array}{l}\text { Power System } \\
\text { Analysis }\end{array}$ & $\begin{array}{l}\text { Protocolos en voz alta } \\
\text { de interpretación léxica }\end{array}$ \\
\hline $\mathbf{8}$ & $\begin{array}{l}\text { Permanent-magnet moving-coil } \\
\text { instruments }\end{array}$ & $\begin{array}{l}\text { Power System } \\
\text { Analysis }\end{array}$ & $\begin{array}{l}\text { Protocolos en voz alta } \\
\text { de interpretación léxica }\end{array}$ \\
\hline $\mathbf{9}$ & Circuit Elements & $\begin{array}{l}\text { Fundamentals of } \\
\text { Electric Circuits }\end{array}$ & $\begin{array}{l}\text { Inferencia de } \\
\text { significado }\end{array}$ \\
\hline
\end{tabular}




\section{Resultados}

En el análisis se considera información cualitativa que describe el proceso seguido en el uso de una determinada estrategia y su efectividad; esta efectividad se asume alta cuando el significado inferido del léxico es correcto para el contexto del texto trabajado en cada uno de los talleres desarrollados por los estudiantes en clase. Esta información se complementa con datos cuantitativos, en términos de frecuencia de uso de las estrategias.

La recolección de la información se hizo en doce semanas, en las que se desarrollaron nueve talleres que permitían identificar las estrategias usadas por los estudiantes para comprender el léxico en los textos de lectura que ofrecía el curso. Los resultados del análisis se agruparon de acuerdo con la modalidad del taller: talleres en modalidad de selección múltiple -textos 1884 Exhibition, Problem Solving y What is Electrical Engineering? (Anexo 1: Texto: What is Electrical Engineering? y Anexo 2: Taller para texto: What is Electrical Engineering?)-; talleres en modalidad de pregunta abierta (textos Nuclear Power Plant y Transmission and Distribution Systems); protocolos en voz alta de interpretación léxica (textos Analog Instruments y Permanent-magnet moving-coil instruments, Parte I y Parte II) (Anexo 3: Rejilla de análisis individual de protocolo en voz alta), y taller de inferencia de significados de palabras (texto Circuit Elements).

El análisis se hizo a cada grupo de talleres de manera independiente (selección múltiple, pregunta abierta, protocolos de voz alta e inferencia de significado y apareamiento con la estrategia usada). Posteriormente, se consolidaron los resultados como se muestra en la Figura 1, Frecuencia de uso de estrategias, y en la Figura 2, Efectividad de las estrategias usadas.

\section{Análisis consolidado}

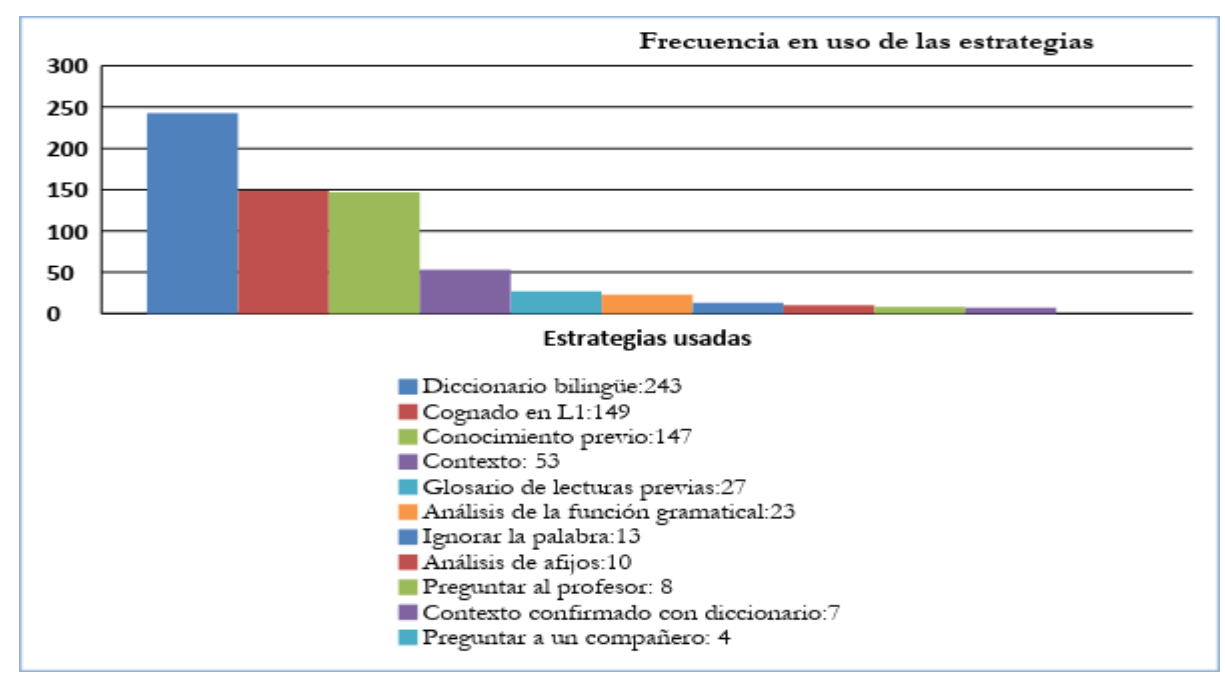

Figura 1. Frecuencia de uso de estrategias 


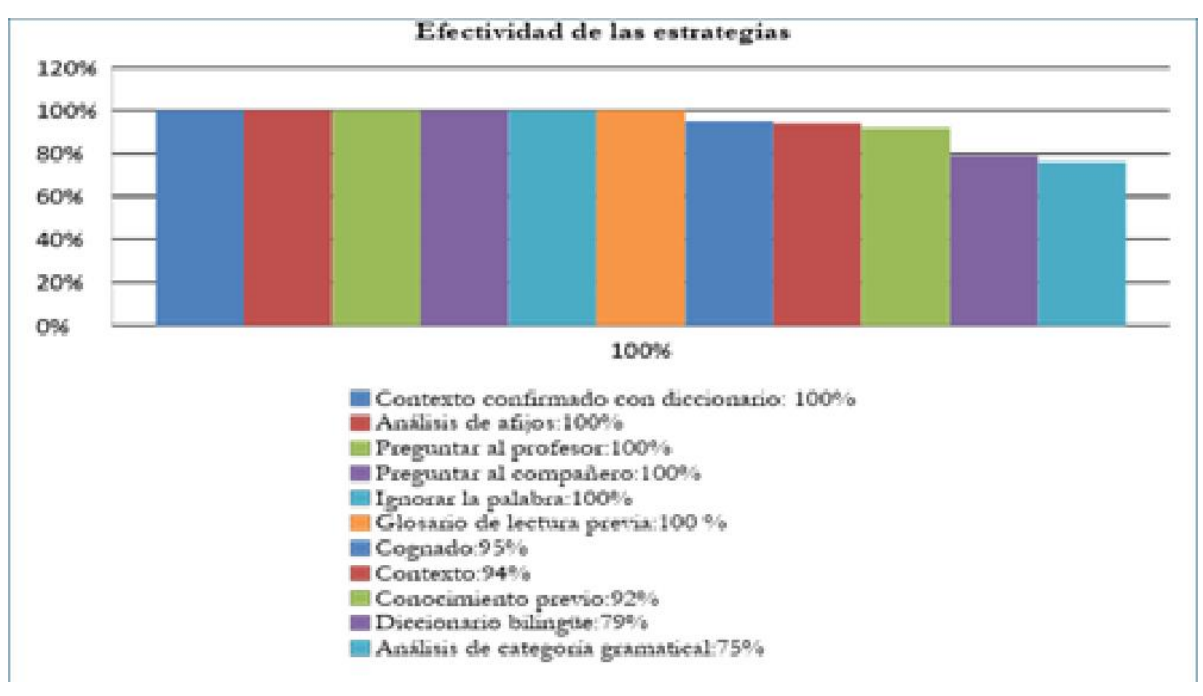

Figura 2. Efectividad de las estrategias usadas

\section{Discusión de los resultados}

El consolidado de la información nos permite identificar una gran variedad de estrategias que los estudiantes usan para comprender nuevo vocabulario en los textos en inglés de su área profesional. Las estrategias pueden ir desde el uso de material de referencia, usar conocimiento previo, identificar cognados, analizar la estructura semántica o gramatical de una palabra hasta pedir ayuda a otra persona, entre otras (ver Figura 1). Dada la variedad encontrada, afirmamos que los estudiantes son conscientes de la existencia de las estrategias léxicas y de su papel en la comprensión de un texto. No obstante, los protocolos en voz alta del uso de estrategias dan cuanta de un uso inconsciente de ellas que genera ineficacia en el propósito lector (ver Figura 2).

La estrategia más empleada por los estudiantes es el uso del Diccionario bilingüe. Esta estrategia es usada con un propósito concreto y, por lo tanto, vale la pena conservarla en el repertorio académico. Sin embargo, su uso es ineficiente en dos aspectos: se ignora la polisemia de los conceptos y se tiene incapacidad de adaptar un significado del diccionario con su rasgo morfológico (sufijo de pasado, pasado participio, gerundio, etc.), de acuerdo con el sentido del texto. La selección indiscriminada de un significado o la incapacidad de inferir uno nuevo muestra la necesidad de comprender la naturaleza lingüística de las palabras en términos morfológicos, sintácticos y semánticos, y este aspecto se podría mejorar en el ejercicio de aula. No se puede partir del supuesto de que los estudiantes de nivel universitario saben manejar el diccionario bilingüe, es importante incluir el manejo de esta estrategia en los programas de los cursos.

Por otro lado, se encuentra que no se hace uso del diccionario monolingüe, a pesar de reconocer que ofrece información contextualizada y conceptos ampliados, muchas veces con ejemplos. El buen uso del diccionario bilingüe requiere ser reforzado 
y podría hacerse en una etapa posterior al uso riguroso del diccionario bilingüe en un primer nivel académico de los cursos de inglés.

La segunda estrategia más usada, y con una alta efectividad, fue Cognados en L1. Se le reconoce como un excelente recurso para inferir significados y para recordar un concepto; en este proceso los estudiantes interrelacionan las dos lenguas: el inglés y el español. Estas relaciones favorecen el aprendizaje, pues la L1 es la base para el abordaje de la L2. Por otro lado, el conocimiento previo de los estudiantes sobre un contenido académico especializado les permite reconocer el cognado y enriquecerlo con conceptos relacionados. Dada la efectividad de esta estrategia, debería ser promovida en los primeros niveles de lengua. Reconocer conceptos relacionados en las dos lenguas genera entusiasmo en los estudiantes, aportando no sólo a la comprensión de los textos sino también a la motivación para el aprendizaje de la lengua extranjera.

En complemento, se observa una correlación entre el nivel de conocimiento técnico de los textos y el reconocimiento de los cognados por parte del estudiante. En cuanto más vocabulario académico, sub-técnico y técnico contiene un texto, mayor número de cognados encontrarán los estudiantes. Dado que los estudiantes de los cursos de ESP están expuestos a textos de carácter académico y científico, es importante hacerlos conscientes de la presencia e importancia del vocabulario técnico en la comprensión de textos de su área profesional.

La tercera estrategia empleada, en términos de frecuencia, fue Conocimiento Previo; esta estrategia se usó con un alto grado de efectividad. Esto confirma que la familiarización con temáticas de la disciplina profesional es un elemento importante por tener en cuenta en el diseño de programas de cursos de Lectura. A su vez, confirma la importancia de establecer relaciones entre el área de inglés y otras asignaturas que cursan los estudiantes en un mismo periodo académico. Un diálogo académico entre los profesores, sin lugar a dudas, da lugar a programas pertinentes para el área profesional y a promover un aprendizaje significativo.

La estrategia Contexto fue la cuarta más usada por los estudiantes. El análisis muestra que cuanto más técnico es el contenido de un texto, menos se usa esta estrategia. En los textos del grupo 2 (Nuclear Plant y Transmission and Distribution Systems) se usó en dos ocasiones mientras que en los del grupo 3 (Analog Instruments, Permanent-magnet moving-coil instruments) no se utilizó. Esto sugiere que los estudiantes encuentran otras estrategias más efectivas para la comprensión de este tipo de textos.

La estrategia Glosario de lecturas previas ocupó el quinto lugar; su uso fue siempre efectivo, pero fue usada por muy pocos estudiantes. De acuerdo con sus observaciones, el vocabulario consultado hace parte del TIER II y TIER III, que recopila vocabulario académico. Este hecho sugiere la posibilidad de incluir listados de conceptos nuevos, como parte del material de clase e inducir el diseño de listados personalizados de conceptos que podrían ser enriquecidos con cada lectura. Este trabajo ayudaría a concientizar a los estudiantes sobre la necesidad de prestar especial atención 
a este tipo de vocabulario, dada su frecuencia de aparición en los textos académicos. Se debe evitar, sin embargo, tener listados de palabras descontextualizadas, por lo que se sugiere hacerlo con definiciones, mapas semánticos, oraciones contextualizadas, etc.

La estrategia Análisis de la categoría gramatical fue la sexta más usada, con una frecuencia relativamente baja y una efectividad aceptable. En las ocasiones en las que se usó de forma efectiva fue, generalmente, para identificar la función del participio pasado en la voz pasiva. Esto se puede atribuir a la alta frecuencia con que los estudiantes están expuestos a esta forma lingüística tan propia de los textos académicos en inglés. Por su importancia en la optimización del uso del diccionario, se considera pertinente hacer consciencia sobre esta relación.

La estrategia Ignorar la palabra fue usada sólo en una ocasión en la que se incluía como estrategia predeterminada en un taller. Muy posiblemente los estudiantes no eran conscientes de que esta opción estratégica permite no interrumpir la lectura. Las palabras ignoradas no eran palabras que aportaran contenido significativo al texto.

El Análisis de afijos se utilizó con poca frecuencia pero siempre con efectividad. Se empleó sólo con sufijos, no con prefijos, y mostró claridad en el significado que el sufijo aporta a la raíz, en términos de sentido y de cambio en la función gramatical. De acuerdo con las observaciones, los estudiantes reconocen haber aprendido a usar los sufijos y ello les ayuda a mejorar las prácticas de clase. Este conocimiento contribuye a reducir la dependencia del diccionario.

Las estrategias sociales de pedir ayuda, en este caso a un compañero o al profesor, se usaron con muy poca frecuencia. En las ocasiones en las que se acudió al profesor, se hizo para consultar unidades léxicas compuestas que aparentemente representan un alto nivel de dificultad para los estudiantes en este momento académico. Teniendo en cuenta que en la práctica laboral futura la interacción con los textos es individual, está bien que los estudiantes no dependan de alguien para resolver dudas de significados durante una lectura sino que desarrollen estrategias propias.

En el proceso de lectura también apareció la unión de dos estrategias, como es el caso de Contexto y Diccionario bilingüe. Se hizo inferencia, a partir de contexto, y se confirmó con el diccionario. Esta integración de estrategias sólo apareció en los primeros talleres; probablemente, la inferencia hecha fue siempre correcta y ello los llevo a tener mayor confianza en lecturas posteriores.

\section{CONCLUSIONES}

\section{Propuesta pedagógica}

Este apartado esboza una propuesta pedagógica que tiene como objetivo promover el desarrollo de estrategias léxicas de aprendizaje para mejorar la comprensión de textos escritos académicos. Como se pudo establecer en el análisis, los estudiantes son 
conscientes de la existencia de estrategias léxicas y de su función en la lectura de textos en inglés. Sin embargo, las estrategias usadas con mayor frecuencia no son siempre las más efectivas y las estrategias usadas con mayor efectividad no son usadas con frecuencia. En el intento de encontrar equilibrios entre la efectividad de las estrategias y la frecuencia en uso de parte de los estudiantes, la propuesta de intervención que aquí se plantea centra su atención en cuatro estrategias de comprensión léxica que se complementan: uso de diccionario bilingüe, definición y revisión de glosario de lecturas previas, análisis de categorías gramaticales de palabra y análisis de afijos.

La estrategia Diccionario bilingüe es incluida por ser la más usada, pero su efectividad puede ser mejorada reconociendo el carácter polisémico de las palabras o sus opciones morfológicas. La estrategia Análisis de afijos, porque es una de las estrategias menos usada por los estudiantes, pero cuando la usan lo hacen con un grado total de efectividad. La estrategia Glosario de lecturas previas por su efectividad en el uso, pero con baja frecuencia. Esta estrategia expone a los estudiantes al vocabulario académico esencial en el contexto universitario. Finalmente, la estrategia Análisis de categoría gramatical, por sus implicaciones en la concientización de la existencia de claves lingüísticas que ayudan a inferir significado de vocabulario nuevo en los textos.

La propuesta sugiere llevar a cabo una instrucción en estrategias léxicas, integrada a los programas de cursos de lectura académica en inglés. Se sugiere, así mismo, que la intervención sea informada; es decir, que los estudiantes conozcan el componente y su papel en el programa. Esta propuesta está estructurada en fases de intervención para cada una de las cuatro estrategias como se presenta en Tabla 2: Uso de Diccionario Bilingüe - Énfasis en elección de significado; Tabla 3: Glosario de lecturas previas; Tabla 4: Análisis de Afijos, y Tabla 5: Análisis de Categoría Gramatical.

Tabla 2. Uso de Diccionario Bilingüe - Énfasis en elección de significado

\begin{tabular}{|l|l|}
\hline FASES DEL & \multicolumn{1}{|c|}{ ESTRATEGIA } \\
\cline { 2 - 3 } MODELO & \multicolumn{1}{|c|}{ Uso de Diccionario Bilingüe - Énfasis en elección de significado } \\
\hline Preparación & $\begin{array}{l}\text { Discusión en clase sobre quién usa el diccionario bilingüe, cuándo lo } \\
\text { usa y dificultades que encuentran en su uso. El profesor hace lista de } \\
\text { las dificultades expresadas por los estudiantes. Si la polisemia no es } \\
\text { mencionada por ellos, el profesor la adiciona a la lista y explica el } \\
\text { concepto. }\end{array}$ \\
\hline & $\begin{array}{l}\text { El profesor modela la estrategia tomando de un texto una palabra } \\
\text { (previamente seleccionada) que tenga más de un significado. } \\
\text { Siguiendo la modalidad de protocolo en voz alta, el profesor describe } \\
\text { oralmente y en voz alta el proceso que sigue en la decisión de qué } \\
\text { hacer con esta palabra. } \\
\text { Ejemplo: la palabra FAST tomada del texto a continuación: (Se sugiere }\end{array}$ \\
\hline
\end{tabular}




\begin{tabular}{|c|c|}
\hline & 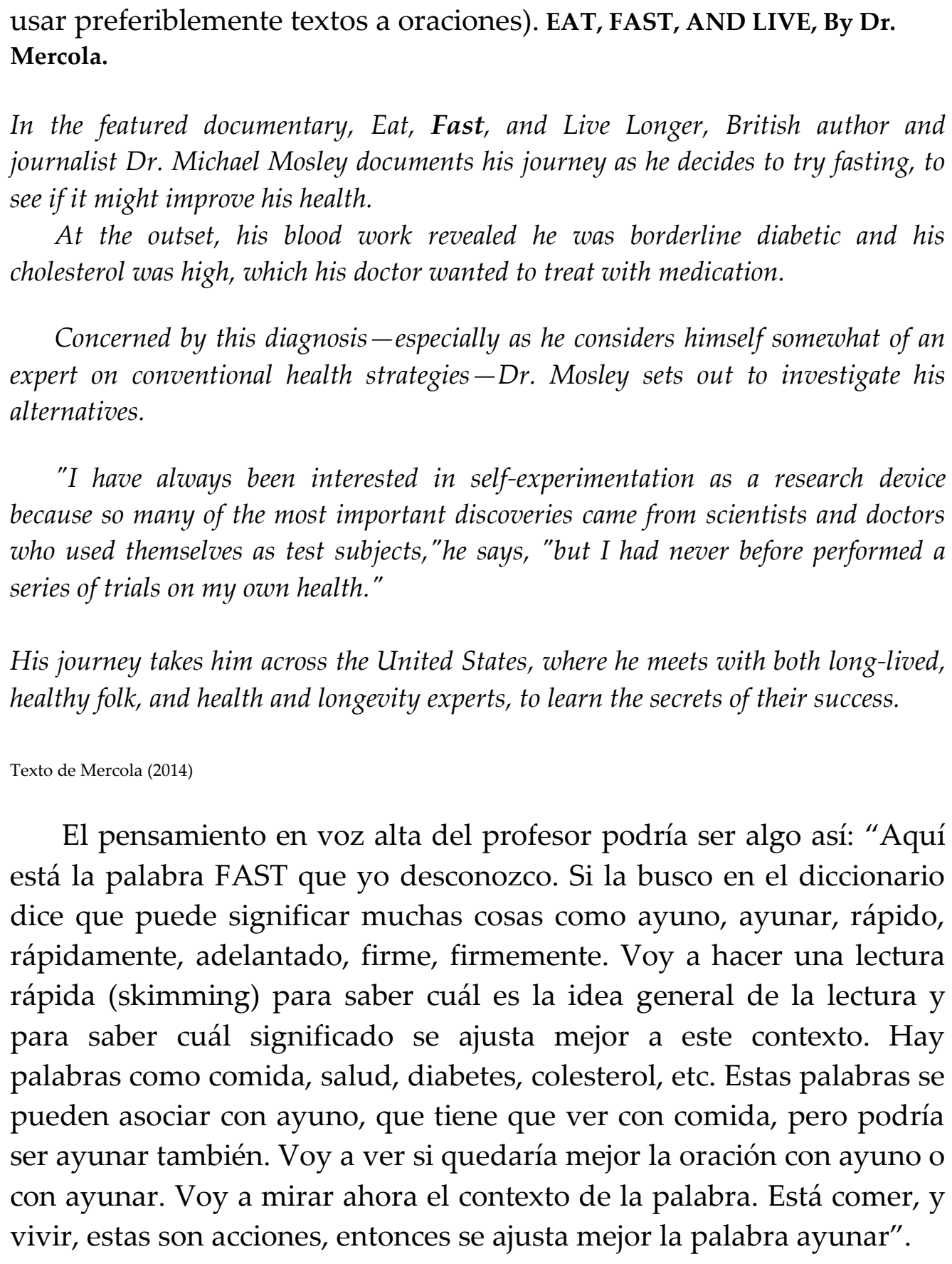 \\
\hline Práctica & $\begin{array}{l}\text { Los estudiantes reciben un texto (se recomienda texto corto para } \\
\text { facilitar comprensión) en el que previamente se haya identificado } \\
\text { vocabulario nuevo con más de un significado. Se les pide a los } \\
\text { estudiantes que consulten la palabra en el diccionario. La actividad de } \\
\text { monitoreo para decidir cuál palabra podría ser la más adecuada se } \\
\text { hace con la participación de todo el grupo; es importante que todos } \\
\text { escuchen diferentes posibilidades hasta decidirse por la mejor opción. } \\
\text { Ejemplo: The delicate look and feel of silk are deceptive. It is a strong, }\end{array}$ \\
\hline
\end{tabular}




\begin{tabular}{|c|c|}
\hline & $\begin{array}{l}\text { natural fiber, ranked in strength with synthetic nylon. To the naked eye, it } \\
\text { appears to be smooth, but under the microscope cultivated silk fiber looks } \\
\text { coarse. It is the coolest of hot-weather fabrics, and it can absorb up to } 30 \\
\text { percent of its weight in moisture without feeling wet. } \\
\text { The word look in the first line of the passage } \\
\text { means: } \\
\text { The word look in the third line of the passage means: }\end{array}$ \\
\hline Evaluación & $\begin{array}{l}\text { Para evaluar la efectividad de la estrategia los estudiantes deben hacer } \\
\text { una lista de las consideraciones que se hicieron para llegar al } \\
\text { significado correcto de la palabra. Por ejemplo: se tuvo en cuenta el } \\
\text { contexto de la palabra, si se ajustaba mejor como verbo o como } \\
\text { sustantivo. Ejercicios de este tipo indudablemente pueden hacer parte } \\
\text { de las actividades de la clase. }\end{array}$ \\
\hline $\begin{array}{l}\text { Actividades } \\
\text { de expansión }\end{array}$ & $\begin{array}{l}\text { Los estudiantes reciben un nuevo texto que deben trabajar de manera } \\
\text { independiente. Se les pide descifrar el significado apropiado de } \\
\text { palabras que tienen más de un significado o pertenecen a más de una } \\
\text { categoría gramatical. }\end{array}$ \\
\hline
\end{tabular}

Tabla 3. Glosario de lecturas previas

\begin{tabular}{|l|l|}
\hline FASES DEL & \multicolumn{1}{c|}{ ESTRATEGIA } \\
\cline { 2 - 3 } MODELO & \multicolumn{1}{c|}{ Glosario de lecturas previas } \\
\hline Preparación & $\begin{array}{l}\text { Se entrega un cuestionario a los estudiantes en el que se les pregunta si al } \\
\text { leer un texto ellos suelen seleccionar y listar el vocabulario nuevo que } \\
\text { encuentran. Si lo hacen, icómo lo registran? Escriben el significado en } \\
\text { español solamente o con ejemplos, la definición en inglés, la explicación } \\
\text { en inglés o en español, escriben sinónimos de la palabra nueva, etc. }\end{array}$ \\
\hline Presentación & $\begin{array}{l}\text { El profesor modela la estrategia presentando visualmente (en proyector) } \\
\text { una lectura en la que se resaltan palabras pertenecientes a los TIERS I y II } \\
\text { del lenguaje académico. Junto con la lectura, presenta una lista en la que } \\
\text { se retoman esas palabras y se explican de diferentes formas: equivalencia } \\
\text { con ejemplo, definición en español, definición en inglés, sinónimos, etc. } \\
\text { En este punto se hace énfasis en que las palabras de la lista son palabras } \\
\text { de corte académico y es común encontrarlas en los textos a los que se } \\
\text { exponen los estudiantes. Ejemplo: Improve (verbo): make better. Ex: I } \\
\text { improved my homework by adding more information. Accuracy (noun): }\end{array}$ \\
\hline Práctica & $\begin{array}{l}\text { A los estudiantes se les entrega un texto de contenido académico. Se les } \\
\text { pide que trabajen en parejas y determinen el vocabulario nuevo para } \\
\text { listarlo y decidir de qué forma podrían ellos recordar mejor su }\end{array}$ \\
\hline
\end{tabular}




\begin{tabular}{|c|c|}
\hline & significado: con ejemplos, con definiciones, con sinónimos, etc. \\
\hline Evaluación & $\begin{array}{l}\text { Se les pide a los estudiantes que autoevalúen el listado del vocabulario } \\
\text { seleccionado, con base en criterios tales como: } \\
\text { 1. La longitud de la lista: muy larga, muy corta, longitud razonable. } \\
\text { 2. El uso de las palabras de la lista: palabras que se usan más en } \\
\text { conversaciones orales que en textos académicos escritos o viceversa. } \\
\text { 3. Las explicaciones de las palabras: sólo traducción, traducción con } \\
\text { ejemplo, definición, sinónimo, etc. }\end{array}$ \\
\hline $\begin{array}{l}\text { Actividades } \\
\text { de Expansión }\end{array}$ & $\begin{array}{l}\text { Se entrega a los estudiantes un texto que deben trabajar de forma } \\
\text { independiente. Se les pide elaborar una lista con el vocabulario nuevo } \\
\text { que consideren importante y útil para lecturas posteriores. Igualmente se } \\
\text { les recomienda usar diferentes formas de comprender y recordar su } \\
\text { significado. Las actividades de expansión se trabajan durante el semestre. }\end{array}$ \\
\hline
\end{tabular}

Tabla 4. Análisis de Afijos

\begin{tabular}{|l|l|}
\hline FASES DEL & \multicolumn{1}{|c|}{ ESTRATEGIA } \\
\cline { 2 - 3 } MODELO & \multicolumn{1}{c|}{ Análisis de Afijos } \\
\hline Preparación & $\begin{array}{l}\text { El profesor dice a los estudiantes que van a trabajar en una estrategia } \\
\text { llamada Análisis de afijos. Les pregunta qué creen ellos que se va } \\
\text { discutir en la lección. Se les pide que ilustren con ejemplos y se toma } \\
\text { nota de sus comentarios. }\end{array}$ \\
\hline & $\begin{array}{l}\text { Se retoma la información correcta que hayan aportado los estudiantes } \\
\text { y se adiciona información nueva sobre los afijos. Se explican los } \\
\text { conceptos de prefijo y de sufijo, y se proveen ejemplos. Se presenta una } \\
\text { lista de prefijos y otra de afijos frecuentes. (No necesariamente todos). } \\
\text { También se presenta con proyector una lectura en la que se resaltan las } \\
\text { palabras que presentan afijos. Se puede decidir presentar prefijos o } \\
\text { sufijos independientemente o ambos en el mismo momento. Se } \\
\text { analizan las palabras desde los afijos y desde el significado que aporta. } \\
\text { Por ejemplo: sufijo "ness": significado que aporta: estado de ser - } \\
\text { vuelve la palabra sustantivo. happy : feliz - happiness: felicidad; sad: } \\
\text { triste - sadness: tristeza. De esta manera se presentan varios ejemplos. }\end{array}$ \\
\hline
\end{tabular}




\begin{tabular}{|c|c|c|c|c|}
\hline \multirow[t]{4}{*}{ Práctica } & \multicolumn{4}{|c|}{$\begin{array}{l}\text { Los estudiantes trabajan en pequeños grupos para apoyarse entre ellos; } \\
\text { reciben una lista dividida en cuatro columnas: en la columna } 1 \text { se } \\
\text { encuentran las palabras sin afijos y con su significado en español. } \\
\text { En la columna } 2 \text { se encuentra el afijo y su significado. En la columna } 3 \\
\text { se encuentran las palabras con el afijo. En la columna } 4 \text { deben escribir } \\
\text { el significado que adquiere la palabra con el afijo. También se puede } \\
\text { dar explicación de la palabra. Se provee un ejemplo de cada afijo. } \\
\text { Ejemplo: }\end{array}$} \\
\hline & Free (libre) & $\begin{array}{l}\text { dom: estado de } \\
\text { ser }\end{array}$ & freedom & Libertad \\
\hline & Wise (sabio) & Dom & Wisedom & sabiduria \\
\hline & $\begin{array}{l}\text { Fever } \\
\text { (fiebre) }\end{array}$ & Ish & Feverish & $\begin{array}{l}\text { Presentar } \\
\text { síntomas } \\
\text { fiebre }\end{array}$ \\
\hline Evaluación & \multicolumn{4}{|c|}{$\begin{array}{l}\text { Se abre un espacio en el que los estudiantes puedan expresar su nivel } \\
\text { de comprensión y pedir aclaración sobre los conceptos que no han } \\
\text { quedado claros o los que necesitan refuerzo. }\end{array}$} \\
\hline $\begin{array}{l}\text { Actividades } \\
\text { de Expansión }\end{array}$ & \multicolumn{4}{|c|}{$\begin{array}{l}\text { Se les entrega una lectura a los estudiantes en la que se pueder } \\
\text { encontrar varias palabras con afijos. Se hacen preguntas de } \\
\text { comprensión que requieran comprensión de algunas de estas palabras, } \\
\text { para exponer así a los estudiantes a los afijos de manera significativa. }\end{array}$} \\
\hline
\end{tabular}

Tabla 5. Análisis de Categoría Gramatical

\begin{tabular}{|c|c|c|c|c|c|c|}
\hline \multirow{2}{*}{$\begin{array}{l}\text { FASES DEL } \\
\text { MODELO }\end{array}$} & \multicolumn{6}{|c|}{ ESTRATEGIA } \\
\hline & \multicolumn{6}{|c|}{ Análisis de Categoría Gramatical } \\
\hline \multirow[t]{4}{*}{ Preparación } & \multicolumn{6}{|c|}{$\begin{array}{l}\text { Identificación del conocimiento previo del estudiante: Se le entrega a } \\
\text { cada estudiante una tarjeta con un texto muy corto en el que se ha } \\
\text { subrayado una palabra. Todas las tarjetas tienen subrayada una } \\
\text { palabra diferente. El docente presenta una tabla en el tablero con la } \\
\text { siguiente información. Se les pide a los estudiantes que } \\
\text { voluntariamente registren en la tabla la palabra que ellos tienen } \\
\text { subrayada en el texto de su tarjeta. } \\
\text { Categorías Gramaticales }\end{array}$} \\
\hline & $\begin{array}{l}\text { noun } \\
(\mathrm{n})\end{array}$ & verb (v) & $\begin{array}{l}\text { adjective } \\
\text { (adj.) }\end{array}$ & $\begin{array}{l}\text { adverb } \\
\text { (adv.) }\end{array}$ & $\begin{array}{l}\text { conjunctio } \\
\mathrm{n} \text { (conj.) }\end{array}$ & \begin{tabular}{|l|} 
prepositional \\
phrase (prep. \\
ph)
\end{tabular} \\
\hline & \multicolumn{6}{|c|}{$\begin{array}{l}\text { De esta forma el docente determina lo que los estudiantes saben sobre } \\
\text { el tema y los vacíos que se deben llenar. }\end{array}$} \\
\hline & \multicolumn{6}{|c|}{ El profesor informa a los estudiantes sobre las características, la } \\
\hline
\end{tabular}




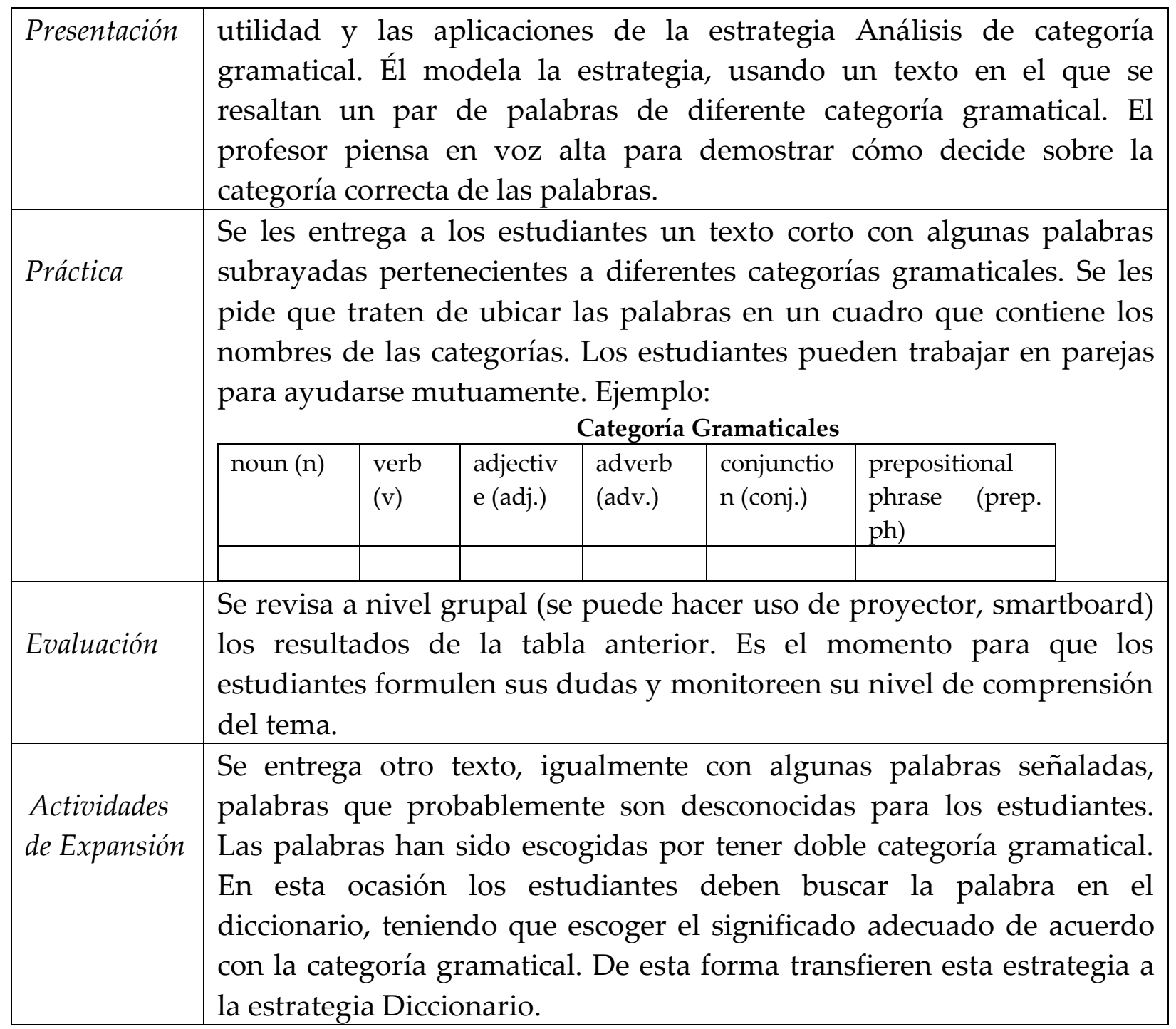

\section{REFERENCIAS}

Akhondi, M., Malayeri, F. A., y Samad, A. A. (2011). How to Teach Expository Text Structure to Facilitate Reading Comprehension. The Reading Teacher, 64(5), 368-372. Recuperado de http://www.literacyhow.com/wp-content/uploads/2015/04/Howto-use-expository-text-structure-to-facilitate-reading-comprehension.pdf.

Barbera, A. (2011). How students use academic vocabulary comprehension strategies in reading history (Tesis de Maestría). Hamline University, Saint Paul, Estados Unidos. Recuperado de https://digitalcommons.hamline.edu/hse_all/454/.

Beck, I., McKeown, M., y Kucan, L. (2013). Bringing Words to Life. Robust Vocabulary Instruction ( $2^{\text {nd }}$ ed.). New York: The Guilford Press. 
Bhatia, V. K. (1993). Analysing Genre: Language Use in Professional Settings. Londres, Inglaterra: Longman.

Brown, P. (2008). A review of the literature on case study research. Canadian Journal for New Scholars in Education 1(1), 1-13. Recuperado de https://journalhosting.ucalgary.ca/index.php/cjnse/article/view/30395.

Carlo, M. S, August, D., McLaughlin, B., Snow, C. E., Dressler, C., Lippman, D. N., ...White, C. E. (2004). Closing the gap: Addressing the vocabulary needs of English-language learners in bilingual and mainstream classrooms. Reading Research Quarterly, 39(2), 188-215. doi: 10.1598/RRQ.39.2.3

Dudley-Evans, T., y St. John, M. (1998). Development in ESP. A multi-disciplinary approach. Cambridge: Cambridge University Press.

Durán Escribano, P. (2001). Funciones de la lectura en la enseñanza del inglés profesional y académico y estrategias de comprensión lectora. Didáctica (Lengua y Literatura), 13, 69-85.

Durán Escribano, P. (2004). Exploring cognition processes in second language acquisition: the case of cognates and false-friends in EST. Ibérica, 7, 87-106.

Gatehouse, K. (2001). Key Issues in English for Specific Purposes (ESP) Curriculum Development. The Internet TESL Journal, 7(10). Recuperado de http://iteslj.org/Articles/Gatehouse-ESP.html.

Hassan, Z., y Abubakr, S. (2015). English Vocabulary Learning Strategies by EFL Learners at University of Sulaimani: A Case Study. International Journal on Studies in English Language and Literature (IJSELL), 3(3), 23-29. Recuperado de https://docplayer.net/47653809-English-vocabulary-learning-strategies-by-efllearners-at-university-of-sulaimani-a-case-study.html.

Hernández, F. (2008). Aprendizaje estratégico: un camino al aprendizaje autorregulado. Cali, Colombia: Universidad del Valle.

Hutchinson, T., y Waters, A. (1987). English for Specific Purposes. A learning-centred approach. Cambridge: Cambridge University Press.

Hyland, K. (2004). Genre and Second Language Writing. Michigan: The University of Michigan Press.

Hyland, K. (2006). English for Academic Purposes: An advanced resource book. New York: Routledge.

Kennedy, C., y Bolitho, R. (1984). English for Specific Purposes. Londres, Inglaterra: MacMillan.

Lakatos, E. (2004). Incidencia de una intervención pedagógica en la enseñanza de dos estrategias metacognitivas para mejorar el nivel de comprensión de lectura en inglés de un grupo de estudiantes universitarios (Tesis de maestría). Universidad del Valle, Cali, Colombia.

McGrath, I. (2002). Material Evaluation and Design for Language Teaching. Edinburgh: Edinburgh University Press. 
Mercola, J. (junio 14 de 2014). How Intermittent Fasting Can Help You Live Healthier, Longer. Mercola.com. Recuperado de http://articles.mercola.com/sites/articles/archive/2014/06/14/intermittent-fastinglongevity.aspx

Nassaji, H. (2003). L2: Vocabulary Learning from Context: Strategies, Knowledge Sources, and Their Relationship with Success in L2 Lexical Inferencing. TESOL Quarterly, 37(4), 645-670. doi: 10.2307/3588216

Nunan, D. (1988). The Learner-Centred Curriculum. Cambridge: Cambridge University Press.

O'Malley, J. M., y Chamot, A. U. (1990). Learning Strategies in Second Language Acquisition. Cambridge: Cambridge University Press.

Ortiz, H. (2011). Estudio descriptivo, explicativo e interpretativo de las estrategias de monitoreo cognitivo usadas por estudiantes universitarios en la comprensión de textos académicos durante la lectura en inglés (Tesis de maestría). Universidad del Valle, Cali, Colombia.

Oxford, R. L. (1990). Language Learning Strategies: What Every Teacher Should Know. Boston: Heinle \& Heinle Publishers.

Robinson, P. (1980). ESP (English for Specific Purposes): The present position. Oxford: Pergamon Press.

Schmitt, N. (1997). Vocabulary Learning Strategies. En N. Schmitt y M. McCarthy (Eds.), Vocabulary: Description, Acquisition and Pedagogy (pp. 199-227). Cambridge: Cambridge University Press.

Swales, J. (1983).Vocabulary work in LSP - A Case of Neglect? En F. Redard y S. Wyler (Eds.), Langues de spécialité: Théorie, application et enseignement. Actes du colloque de la Commission interuniversitaire suisse de linguistique appliquée (Bulletin CILA) (pp. 2133).

Recuperado

de http://doc.rero.ch/record/11876/files/bulletin_vals_asla_1983_037.pdf.

Trimble, L. (1985). English for Science and Technology, A discourse approach. Cambridge: Cambridge University Press.

Wang, Q. (2011). Lexical Inferencing Strategies for Dealing with Unknown Words in Reading-A Contrastive Study between Filipino Graduate Students and Chinese Graduate Students. Journal of Language Teaching and Research, 2(2), 302-313. doi: 10.4304/jltr.2.2.302-313

Yin, H. (2010). A study of L2 Vocabulary Learning Strategies (Tesis de Maestria). Kristianstad University, Kristianstad, Suecia. Recuperado de http://www.divaportal.org/smash/get/diva2:326994/FULLTEXT01.pdf

Zarrati, Z., Nambiar R. M. K., y Tg. Mohamad Maasum, T. N. R. (2014). The Importance of Text Structure Awareness in Promoting Strategic Reading among EFL Readers. Procedia - Social and Behavioral Sciences, 118, 537-544. doi: 10.1016/j.sbspro.2014.02.073 


\section{SOBRE LAS AUTORAS}

\section{Martha Isabel Hernández Pastuzano}

Título de maestría en Lingüística y Español; áreas de desempeño académico: enseñanza del inglés y estrategias de aprendizaje; área de desempeño laboral: Desarrollo Infantil (asistente facilitadora), en Renfrew Educational Services en Calgary, Canadá.

Correo electrónico: mcastano@renfreweducation.org

\section{Fanny Hernández Gaviria}

Profesora titular de la Universidad del Valle con título de maestría en Lingüística y Español; áreas de desempeño académico: Lingüística Aplicada y formación en investigación.

Correo electrónico: fanny.hernandez@correounivalle.edu.co 


\section{ANEXOS}

\section{Anexo 1: Texto What is Electrical Engineering?}

Anexo 3

\section{What is Electrical Engineering?}

1 The professional activities of electrical engineers directly affect the everyday lives of most of the world's population. They are responsible for the design and development of radio and television

5 transmitters and receivers, telephone networks and switching systems, computer systems, and electric power generation and distribution. Within the broad scope of these systems, the electrical engineer is concerned with a challenging and diverse array of

10. design and development problems.
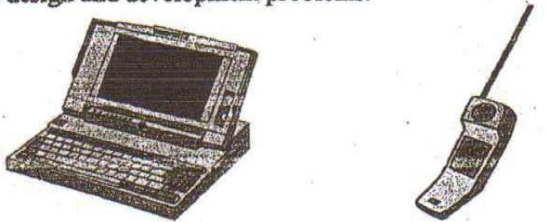

- Electrical engineers design miniature semiconductor - integrated circuits that contain millions of elementary devices. Electrical engineers also create systems for automatically controlling mechanical

15 devices and a variety of processes. Electrical engineers are responsible for the design of satellite communication links as well as patient monitoring systems for hospitals. The development of the microprocessor has expanded the opportunities for

20 electrical engineers to improve the design of familiar products since these devices are now incorporated in automobiles, consumer and office products, entertainment systems, and a vast variety of test and measurement instruments and machine tools.

25 - Students who earn a B.S.E. degree in Electrical Engmeering will be involved in a variety of electrical and electronic problems in the course of their careers. To ensure the necessary breadth of

30 knowledge, the ASU Electrical Engineering curriculum includes basic (core) engineering courses and courses in networks and electronic circuits, electro-

35 magnetic fields and waves, signal processing, microprocessors, communication and control systems, solid state electronics, electrical power systems, and other special courses. The following describes our major areas of emphasis.

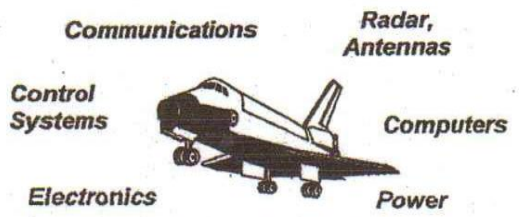

Digital Signal Processing

Solid State Electronics is the study of the behavior 40 of solids. It deals mainly with the behavior of semiconductor materials and devices.' Solid State Electronics is also concerned with other solids, such as insulators and conductors, but semiconductors)are by far the most important. Semiconductors are very 45 important today because they are the building blocks of integrated circuits and other electronic circuits. The most important semiconiductor is silicon, because it is the basis. of most integrated circuits.

The circuits that make computers work are made of 50 semiconductors. They do the calculations, the memory function, and drive the display screen. Integrated circuits are the heart of electronic watches, where they direct the oscillating quartz

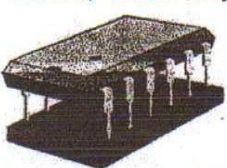
crystal that controls the time. 55 Semiconductor chips control television sets and radios. Semiconductors other than silicon can be made to emit light and are used today in the 60 brake safety lights seen in the spoiler of certain cars. The lasers that are used to detect the sound in compact disk players are made of semiconductors, as are the light emitters and light detectors in fiber optic communication systems. Large semiconductor 65 devices are used in the transmission of power where they convert direct current to alternating current and vice versa. Semiconductors are utilized to charge the battery in a car and control many other functions in a car, from the crash impact detector for releasing 70 air bags, to chips that control the automated braking system. 


\section{Anexo 2: Taller para Texto What is Electrical Engineering?}

Read the passage 'What is Electrical Engineering' and answer the questions regarding the underlined words. Explain how you figured out the meaning you chose. If more space is needed to explain, use the back of this sheet.

1. The word network (line 5) in the passage is closest in meaning to
a. system
c. necessity
b. pole
d. web

Strategy used:

2. The word array (line 9) in the passage is closest in meaning to
a. display
c. tool
b. arrangement
d. variety

Strategy used:

3. The word devices (line 15) in the passage is closest in meaning to
a. utensils
c. designs
b. plans
d. images

Strategy used:

4. The word incorporated (line 21) in the passage is closest in meaning to
a. included
c. integrated
b. excluded
d. designed

Strategy used:

5. The word ensure (line 28) in the passage is closest in meaning to
a. continue
c. create
b. guarantee
d. develop 
Strategy used:

6. The word wave (line 35) in the passage is closest in meaning to
a. signal
c. vibration (physics)
b. undulate
d. flow

Strategy used:

7. The word deals (line 41) in the passage is closest in meaning to
a. examines
c. distributes
b. agreements
d. pacts

Strategy used:

8. The word basis (line 49) in the passage is closest in meaning to
a. remain
c. foundation
b. control
d. explanation

Strategy used:

9. The word emit (line 59) in the passage is closest in meaning to
a. discharge
c. change
b. stop
d. extinguish

Strategy used:

10. The word current (line 67) in the passage is closest in meaning to
a. present
c. tendency
b. flow (of electricity)
d. stream

Strategy used: 
Anexo 3: Rejilla de análisis individual de protocolo en voz alta

\begin{tabular}{|l|l|l|l|l|}
\hline Concepto descifrado & Estrategia usada & Equivalencia & \multicolumn{2}{|l|}{$\begin{array}{l}\text { Evaluación de la } \\
\text { respuesta }\end{array}$} \\
\cline { 4 - 6 } & & & Efectiva & No efectiva \\
\hline electromechanic & cognado en L1 & electromecánicos & & \\
\hline electric & cognado en L1 & eléctrica & & \\
\hline conversion & cognado en L1 & conversión & & \\
\hline mechanic & cognado en L1 & mecánico & & \\
\hline coil & $\begin{array}{l}\text { uso de conocimiento } \\
\text { previo }\end{array}$ & $\begin{array}{l}\text { bobina - } \\
\text { devanado }\end{array}$ & & \\
\hline permanent-magnet & $\begin{array}{l}\text { uso de conocimiento } \\
\text { previo }\end{array}$ & $\begin{array}{l}\text { magneto } \\
\text { permanente }\end{array}$ & & \\
\hline portable & diccionario bilingüe & Portátil & & \\
\hline performance & diccionario bilingüe & Funcionamiento & & \\
\hline found & contexto & Encontrados & & \\
\hline
\end{tabular}

